\title{
Vague Composition Without Vague Existence
}

CHAD CARMICHAEL

Indiana University-Purdue University Indianapolis

\begin{abstract}
David Lewis (1986) criticizes moderate views of composition on the grounds that a restriction on composition must be vague, and vague composition leads, via a precisificational theory of vagueness, to an absurd vagueness of existence. I show how to resist this argument. Unlike the usual resistance, however, I do not jettison precisificational views of vagueness. Instead, I blur the connection between composition and existence that Lewis assumes. On the resulting view, in troublesome cases of vague composition, there is an object, which definitely exists, about which it is vague whether the relevant borderline parts compose it.
\end{abstract}

I think that there are objects - my chair, for example - that are composed of several material objects. But I deny that there is anything composed of my nose and the Eiffel tower. So I'm a moderate about composition, since I think that there is a restriction on composition, but not a total restriction. Furthermore, I think that the restriction is more or less what you would intuitively take it to be: noses are in; towers are in; tower-noses are out. 
David Lewis (1986) claims that this view is committed to the possibility of an incoherent sort of vague existence, and he concludes that composition is in fact unrestricted. ${ }^{1}$ As he puts it:

The question whether composition takes place in a given case, whether a given class does or does not have a mereological sum, can be stated in a part of language where nothing is vague. Therefore it cannot have a vague answer. There is such a thing as the sum, or there isn't. It cannot be said that, because the desiderata for composition are satisfied to a borderline degree, there sort of is and sort of isn't. What is this thing such that it sort of is so, and sort of isn't, that there is any such thing? No restriction on composition can be vague. But unless it is vague, it cannot fit the intuitive desiderata. So no restriction on composition can serve the intuitions that motivate it. So restriction would be gratuitous. (213)

To say that "there sort of is and sort of isn't" a sum of the relevant class is just to say

(1) It is vague whether there is something that is the sum of that class.

To say that there is a thing of which "it sort of is so, and sort of isn't, that there is any such thing" is to say

(2) There is something of which it is vague whether there is any such thing.

Thesis (2) is of course absurd. If something is red, then it is not vague whether it is red. Similarly, if there is such a thing as $x$, then it is not vague whether there is any such a thing as $\mathrm{x}$. Lewis thinks that (1) entails the absurd (2). Why does Lewis think this?

\footnotetext{
$1 \quad$ Also see Ted Sider $(2001,120$ - 32) for an elaboration and defense of the argument. Sider extends the argument to support four-dimensionalism as well as unrestricted composition. The argument has received a great deal of attention in the literature. For example, see van Inwagen (1990, section 19), Markosian (1998a; 2004), Hawley (2002), Koslicki (2003), Merricks (2005), Hawthorne (2006), Nolan (2006), and Smith (2006).
} 
An example helps. Consider a sparsely populated world containing some mereological simples that are arranged in such a way that, on a moderate view, it is vague whether they compose anything. (Perhaps they are not quite unified enough to definitely compose a cloud of simples on a moderate view.) Call this the case of the cloudishly arranged simples. Lewis thinks that whether there is anything in addition to the simples in this case depends on whether composition has occurred. As a result, Lewis thinks, since the moderate accepts (1), and so holds that it is vague whether composition has occurred, he must also agree that it is vague whether there is anything in addition to the simples. But Lewis holds that, if it is vague whether there is anything in addition to the simples, then there is something about which it is vague whether it is a thing in addition to the simples. (This is an instance of the principle $\nabla \exists \mathrm{x} \phi \rightarrow \exists \mathrm{x} \nabla \phi$, where ' $\nabla$ ' is read 'it is vague whether'.) But that is to say that there is a thing about which it is vague whether there is any such thing, which is the absurd (2).

So the argument is that (1) entails (2) in this sort of case. Specifically, this is a case in which the moderate will say that none of the candidate composers is definitely a part of a composite for which they (jointly) are candidate composers. For example, if it is vague whether a board and a stump compose a table, then neither the board nor the stump is definitely part of a table for which they are candidate composers. Similarly, none of the simples is definitely a part of any object for which they are candidate composers. I call these homogeneous cases of borderline composition, since the candidate composers uniformly fail to definitely be a part of a composite for which they (jointly) are candidate composers. By contrast, consider some objects - this rock on the summit, that rock in the penumbral region, and lots of other stuff — which are (jointly) candidates for composing Kilimanjaro. The rock on the summit is definitely a part of Kilimanjaro, while the rock in the penumbral region is not definitely a part of it. In this sense, these objects are a heterogeneous case of borderline composition.

Why must Lewis focus on the homogeneous cases? Suppose that some case is heterogeneous. Then there must be an object among the candidate composers that is definitely a part of the composite for which the objects are (jointly) candidate composers. But then that composite object definitely exists, and it's existence clearly does not hinge on how the vagueness is resolved concerning whether the candidates compose anything. So it is only in homogeneous cases of borderline composition that it is at all plausible to say that the existence of a further object, in addition to the relevant candidates, depends on whether they compose 
anything. Lewis's argument, which requires such a claim, must therefore focus on cases that the moderate will regard as homogeneous.

Moderates have typically responded to Lewis's argument by denying the principle $\nabla \exists \mathrm{x} \phi \rightarrow \exists \mathrm{x} \nabla \phi$, as well as the specific instance of it to which Lewis has to appeal. ${ }^{2}$ Moderates who respond in this way claim that denying this principle amounts to embracing a moderate kind of vague existence distinct from the absurd extreme sort of vague existence expressed by (2).

The main problem with this reply is that it is incompatible with the widely held view that vagueness requires precisifications. According to this sort of view, whenever it is vague whether $\phi$, there must be an expression in $\phi$ having multiple admissible precisifications - precise candidate extensions among which we are in some sense undecided. This sort of view, which Lewis was assuming to be correct, apparently requires the principle $\nabla \exists x \phi \rightarrow \exists x \nabla \phi$. To see why, suppose that a precisificational theory is true, and suppose that we have $\nabla \exists x \phi$. In that case, there must be some expression in $\exists x \phi$ with multiple candidate precisifications. Suppose that all such expressions are in $\phi$. In that case, there are objects that are in some but not all candidate extensions for $\phi$. But, on a precisificational theory, that is just equivalent to saying that $\exists x \nabla \phi$, which is the consequent of the principle. As a result, if the principle fails, then it must be due to vagueness of the existential quantifier itself. So the existential quantifier would have multiple candidate extensions. But this is impossible. Since our intention in the present context is to quantify absolutely generally, the intended extension of the quantifier would definitely be the union of the candidates. ${ }^{3}$ It would thus have a definite extension after all. The present sort of response to Lewis's argument is for this reason inconsistent with precisificational theories of vagueness.

I want to suggest a different reply-one that is consistent with both a precisificational account of vagueness and a moderate view of composition. The reply preserves the principle $\nabla \exists x \phi \rightarrow \exists x \nabla \phi$. As a result, in the cloudishly arranged simples case, I say that, since it is vague whether the class of simples has a sum, there is something - a proto-cloud, as I call it - about which it is vague whether it is the sum of the class. This object, the proto-cloud, definitely exists. But it is vague whether it is composed of the simples, and vague whether it has the simples as parts. The view therefore entails the vagueness of 'composition' (and 'part'), contrary to Lewis. But this is not problematic in the way that Lewis

\footnotetext{
For example, see Peter Van Inwagen (ibid., sec. 19) and Katherine Hawley (2002).
}

3 See Sider $(2001,128-9)$ for further discussion of this point. 
thought. For Lewis rejected the vagueness of 'composition' in order to avoid the vagueness of 'exists' (and 'there is'). But, according to the present reply, we may avoid the vagueness of 'exists' and 'there is' in another way: by refusing to assert the premise that whether there is a thing in addition to the simples depends on whether the simples compose anything. According to the resulting view, the boundary between non-being and being is not the boundary between noncomposition and composition. Rather, the boundary between non-being and being is the outer penumbral boundary for composition: the boundary between superdefinite cases of non-composition and everything else, where a super-definite case of non-composition is one such as the case of my nose and the Eiffel Tower-one in which it is definitely ... definitely not the case that composition occurs, for any number of iterations of 'definitely'. (I will say more about this boundary below.)

This view says that there is an object in addition to the cloudishly arranged simples - a proto-cloud. I have found it helpful, in explaining the view, to answer some basic questions about the persistence conditions and location of protoclouds. This is what I will endeavor to do in the rest of the present section. Then, in the subsequent sections, I shall attempt to answer three objections to the view.

Can the proto-cloud persist through condensation into a (definite) cloud? The answer is that, just as a proto-stellar cloud can become a star, a proto-cloud can become a cloud. The alternative, that the proto-cloud is at some point destroyed, and replaced with a cloud, seems implausible. Furthermore, this view would give rise to vague existence, since it would be vague when the replacement occurred, and so vague when the definite cloud came into existence. ${ }^{4}$

What is the spatial location of the proto-cloud ${ }^{5}$ Call this the location question. Since it is vague whether the proto-cloud is composed of the simples, we might expect it to be located in a region $\mathrm{R}$ about which it is vague whether $\mathrm{R}$ is

4 Interestingly, if proto-clouds can become clouds, then there seem to be counterexamples to the truth of many widely accepted scientific essentialist claims (i.e., claims of a posteriori necessity), such as the claim that, given a mass $\mathrm{m}$ of water, $\mathrm{m}$ necessarily has hydrogen atoms as parts. For, if we can find a case in which we have a proto-water-mass, that object will come to form a mass of water with which it is identical, even though we could not say of the protomass that it has hydrogen atoms as parts.

5 By 'spatial location' I mean what Parsons (2007) means by 'exact spatial location': L is the exact spatial location of $\mathrm{x}$ iff no part of $\mathrm{L}$ is free of $\mathrm{x}$ and every spatial region that does not overlap L is completely free of $\mathrm{x}$. So my body is exactly located in a region shaped like a body, and has never been exactly located in a region shaped like an office, or in a region shaped like an arm. 
composed of the locations of the simples. This, however, requires that regions of space can have borderline parts, and, in particular, that there is a region of space about which it is vague whether it is composed of the locations of the simples. It would also follow that composition is restricted for regions of space. For suppose that it is vague whether $\mathrm{R}$ is composed of the locations of the simples, and suppose that composition is unrestricted for regions of space. Then some region $\mathrm{R}^{*}$ is composed of the locations of the simples. $\mathrm{R}$ and $\mathrm{R}^{*}$ are distinct: $\mathrm{R}^{*}$, unlike $\mathrm{R}$, is definitely composed of the locations of the simples. Distinct regions of space never have the same location. So $\mathrm{R}$ and $\mathrm{R} *$ do not have the same location. But, if $\mathrm{R}$ is composed of the locations of the simples, then it does have the same location as $\mathrm{R}^{*}$. So $\mathrm{R}$ is not composed of the locations of the simples, contrary to our supposition that it is vague whether it is composed of the locations of the simples. Proponents of this view, then, cannot accept that there is such a region of space as $\mathrm{R}^{*}$. They cannot accept, that is, that there is a region composed of the locations of the simples. As a result, they cannot accept unrestricted composition for regions of space. That seems to me a significant drawback, since unrestricted composition is plausible for regions of space (certainly more plausible than it is for objects like noses and towers). ${ }^{6}$ Furthermore, as we will see below (in the discussion of objection 1), this view does not fit well with my theory about proto-clouds. I will therefore set this answer to the location question aside. ${ }^{7}$

A second answer to the location question is that it is vague whether there is a location L such that the proto-cloud is located at L. This answer can also be ruled out. For every cloud is definitely a material object. And, for every definite material object $\mathrm{x}$, it is definitely the case that there is a location at which $\mathrm{x}$ is located. So, on the second answer to the location question, it follows that the proto-cloud is not a cloud, contrary to my hypothesis that it is vague whether it is a cloud.

A third possible answer to the location question is that although the proto-cloud definitely has some location or other, there is no particular location about which it is definitely the case that the proto-cloud is located in that location. This view seems right for certain objects. For example, it seems true of Kilimanjaro: it is definitely the case that Kilimanjaro has some location or other, even though

6 Even van Inwagen (1981,1990), who denies the existence of almost all composite objects, appears to accept that there is a region of space corresponding to any set of spatial points.

7 Thanks to an anonymous referee for drawing my attention to this answer to the location question. And thanks to George Bealer for discussion of the argument of this paragraph. 
(because of the vagueness of Kilimanjaro's extent) there is no particular location of which it is definitely true that Kilimanjaro has that location. Here the idea is that some objects among some candidate composers of Kilimanjaro have more of a claim than others to being parts of Kilimanjaro. So some admissible precisifications count more candidates as parts, while some precisifications count fewer of the candidates as parts. As a result, some admissible precisifications count Kilimanjaro a little larger, some a little smaller. So it is vague which of many locations is the location of Kilimanjaro.

Whether the proto-cloud is like Kilimanjaro in this respect depends on how we conceive of the case. If each of the simples has an equal claim to being a part of something, then any admissible precisification that allows one to be a part will allow them all to be parts. The admissible precisifications would then not generate a plurality of candidate locations for the proto-cloud. Rather, there would be just one candidate: the sum of the locations of the simples. On the other hand, if we conceive of the case so that, say, the simples near the center of the protocloud are more unified than the simples around the edge (but still not unified enough to definitely compose anything) then the simples near the center might have a greater claim to parthood than the simples around the edge. In that case, there might well be multiple candidate locations for the proto-cloud, and the present answer to the location question - the answer which says that although the proto-cloud definitely has some location or other, there is no particular location about which it is definitely the case that the proto-cloud is located in that location-would be correct.

I thus favor a fourth answer to the location question: that the location of the proto-cloud is the sum of the locations of some of the simples. Then, depending on the case, the proto-cloud might be definitely located in the sum of the locations of all the simples, or it might be that there is no single sum of simple-locations of which it is definitely true that the proto-cloud is located in that location. It follows from this answer to the location question that being located in a sub-region of a thing's location does not entail being a part of that thing. For, on this view, it can happen that a simple $\mathrm{S}$ is definitely located in a sub-region of the proto-cloud's location, even though $\mathrm{S}$ is not definitely a part of the proto-cloud. Thus, I distinguish mereological overlap, which involves sharing parts, from spatiotemporal overlap, which involves mereological overlap of spatiotemporal locations. ${ }^{8}$ The proto-cloud overlaps the simples spatiotemporally, but does not

8 These must be distinguished by "two-thingers" about the lump and the statue. For if the lump and statue are distinct, then the arm seems to be a part of the statue but not the lump, since 
definitely overlap them mereologically. This will be important in my reply to the first of the three objections which I will now address.

\section{First Objection: Simplicity}

In the cloudishly arranged simples case, is the proto-cloud itself a simple? If we define 'simple' as usual, so that it means 'lacks proper parts', then it is vague whether the proto-cloud is a simple, since it is vague whether it has any proper parts. But this is problematic. For, given our answer to the location question, the proto-cloud is located in a discontinuous region: some sum of the locations of the simples. And simples are definitely not located in discontinuous regions. So the proto-cloud would definitely not be a simple, contradicting what I said above, that it would be vague whether it is a simple. In this way, then, it might appear that my response to Lewis clashes with our intuitions about simplicity. This is the first objection.

One reply to this objection is to define 'simple' as meaning 'definitely lacks proper parts' instead of just 'lacks proper parts'. Then we can say that the protocloud is definitely not simple (since it definitely does not definitely lack proper parts) while it is also vague whether it is composite. This is a natural way of defining 'simple' on the view I am suggesting. Alternatively, we might admit that 'simple' means 'lacks proper parts' but that we easily confuse intuitions about simplicity with intuitions about definite simplicity, since indefinite simples are an exotic case. Either way, the view I am suggesting can handle these intuitions about simplicity.

Of course, one might attempt to press the objection without appeal to the word 'simple', and thus without any appeal to intuitions about simplicity. One might assert that, if an object is located in a discontinuous region, then it definitely has proper parts. But this claim simply begs the question. It is precisely my view that there can be an object which is located in a discontinuous region, but about which it is vague whether it has proper parts. An argument would be required to establish that any object so located must definitely have proper parts.

extreme deformation of the arm seems to destroy the arm without destroying any part of the lump. Proponents of the possibility of interpenetrating matter (e.g., Sider 2001, p. 141, and McDaniel 2007a) are also committed to this distinction between spatiotemporal overlap and mereological overlap. And proponents of extended simples (e.g., Markosian 1998, Scala 2002, Sider 2007, Parsons op. cit., and McDaniel 2007b) are committed to the idea that some matter can occupy a sub-region of an extended simple without being part of that simple. This seems close to the distinction between two kinds of overlap. 
Perhaps the following argument is in the offing:

1. Something that is located in a discontinuous region is partly located in each continuous sub-region thereof.

2. Anything partly located in a given region has a part that is located in that region.

3. Thus, anything located in a discontinuous region has a part.

The argument equivocates between two senses of 'partly located'. In one sense, to be partly located in a region $\mathrm{R}$ is to be located in a region of which $\mathrm{R}$ is a subregion. In another sense, to be partly located in a region is to have a part located in that region. Since my view distinguishes mereological and spatial overlap, I claim that these two senses are not definitely necessarily equivalent. When 'partly located' is understood in the first sense throughout, I refuse to assert the second premise, since the proto-cloud is partly located (in this sense) in various regions at which it has no definite part. When 'partly located' is understood in the second sense, on the other hand, I refuse to assert the first premise.

Note that, if we accept the first answer to the location question-the answer which says that the proto-cloud is located in a (discontinuous) region about which it is vague whether that region is composed of the locations of the simples - then we cannot block this argument in this way. For it will no longer be true that the proto-cloud is located in a region of which the locations of the simples are proper sub-regions. Rather, it will be vague whether those locations are proper subregions of the region in which the proto-cloud is located. And so we will no longer be able to say, as required by my response to the argument, that there is a sense in which the proto-cloud is partly located in those regions. Thus, the first answer to the location question leaves us with no adequate answer to this argument, which is one reason that I prefer my answer to the location question.

Philosophers of a Humean bent will perhaps agree with me in rejecting the necessary equivalence of these two senses of 'partly located', as they might be suspicious of the resulting necessary connection between the mereological structure of objects and the mereological structure of the regions that they occupy. That is, they might be suspicious of the idea that there is a necessary connection between having a certain mereological structure, on the one hand, and being located in a region with that mereological structure, on the other. Indeed, several such philosophers have argued for the possibility of extended simples on the grounds that there is no such necessary connection between the structures of 
objects and their locations. ${ }^{9}$ I do not have such Humean inclinations. But I will take allies where I can find them, even Humeans.

\section{Second Objection: Sharp Cut-off}

Suppose some simples slowly coalesce, starting from a state in which a moderate would say that it is definitely the case that there is nothing that the simples compose. At some point, the simples become unified enough that a moderate feels comfortable saying that there is something they compose. Noticing that the coalescence forms a sorites series, someone might argue as follows.

Either there is a sharp boundary at which something in addition to the simples pops into existence, or else there is no such sharp boundary. But a sharp boundary here is intuitively problematic. And yet, if there is no sharp boundary, then we have accepted vague existence after all, so it will be vague whether there is an additional object at some stage in the coalescence, and so, by the principle $\nabla \exists x \phi \rightarrow \exists x \nabla \phi$, there will be extreme vague existence, which we had intended to avoid.

I have said that the line between non-being and being is the line between superdefinite cases of non-composition and the rest of the cases. According to the most plausible precisificational views of vagueness, this boundary is sharp. To see this, define an operator $D^{*}$, following Williamson (1994, p. 160), like this:

$$
\text { D*A } \leftrightarrow \text { DA \& DDA \& DDDA \& } \ldots
$$

Then a case which is super-definite with respect to A is one in which this holds:

$$
\text { D*A }
$$

Williamson points out that the definition of $\mathrm{D}^{*}$ ensures the following:

$$
\mathrm{D}^{*} \mathrm{~A} \rightarrow \mathrm{DD} * \mathrm{~A}
$$

\footnotetext{
$9 \quad$ See Sider (2007), McDaniel (2007b).
} 
That is, every super-definite case in which A is definitely super-definite: there can be no vagueness concerning super-definiteness. ${ }^{10}$ As a result, precisificational accounts are committed to a sharp line between super-definite cases and the rest.

My view is that this is the line between being and non-being in a sorites series for composition. In other words, my view identifies the line between being and non-being with a sharp line to which the precisificationalist is antecedently committed. If the sharpness of this line is problematic, then it is a problem for precisificational views of vagueness, in which case Lewis's attack on moderate composition fails, since his attack requires such a view of vagueness.

Williamson suggests (p. 160) that the precisificationalist might to avoid the sharpness induced by $D^{*}$ by using a further operator, $D$ !, to capture the vagueness of D*. For, given D!, we can say that the principle $\mathrm{D}^{*} \mathrm{~A} \rightarrow \mathrm{D}$ ! $\mathrm{D}^{*} \mathrm{~A}$ fails. Iterating the process, we would need to introduce a hierarchy of operators: D!!, D!!!, etc.. This is because, for each operator, we would need a further operator in terms of which we can capture the vagueness of the last one.

Williamson's suggestion is problematic on several counts. I'll mention two. First, it is difficult to see why the failure of $D^{*} A \rightarrow D ! D^{*} A$ captures a sense in which $\mathrm{D}^{*}$ is vague, especially given that $\mathrm{D}^{*} \mathrm{~A} \rightarrow \mathrm{DD}^{*} \mathrm{~A}$ remains true. The distinction between different operators seems to be a mere logician's trick without any intuitive basis. Second, as Varzi (2007) points out, there are intelligible uses of 'definitely' that do not fit into the posited hierarchy of operators. For example, someone might say that everything God believes is definitely true. If, as the present reply has it, there is no one sense of 'definitely' in terms of which we can express all the facts about vagueness, then (given that God believes all the truths about vagueness) it is impossible to interpret this claim. Since the claim is perfectly intelligible, then, there must be one such sense of 'definitely'. So it seems that Williamson's first suggestion for avoiding the sharpness of $\mathrm{D}^{*}$ is not promising.

Williamson also suggests (p. 160-1) that the precisificationalist might attempt to avoid sharp lines by denying that almost anything is ever super-definitely true. But this is even less plausible than the first suggestion. For consider an utterly

10 The proof is simple. Suppose D*A. Then, by definition of $\mathrm{D}^{*}$, we have DA \& DDA \& ... That is, each conjunct of A \& DA \& ... is definitely true. Thus, every conjunct in DA \& DDA $\& \ldots$ is definitely true. So each conjunct of this conjunction is true in every admissible precisification. A conjunction is true in a precisification if its conjuncts are true in that precisification. So the conjunction itself is true in every admissible precisifiction. So our conjunction is definitely true: $\mathrm{D}(\mathrm{DA} \& \mathrm{DDA} \& \ldots)$. By definition of $\mathrm{D}^{*}$, this is equivalent to DD*A. 
hairless man. It is difficult to see how the sentence 'That man is bald' could be rendered false by prefixing it with any number of occurrences of $\mathrm{D}$. The man is as bald as it is possible or conceivable for anything to be!

Rather than accepting Williamson's suggestion, the precisificationalist should instead simply embrace the result that $\mathrm{D}^{*}$ imposes a sharp line (that is in principle unknowable) on a standard sorites series. Intuitively, there is no sharp line between (say) the red and the not red in a standard sorites series for 'red'. And, intuitively, there is no sharp line between the definitely red and the not definitely red. But, when we iterate the 'definitely' operator a few more times, I think most of us will admit that we lose our grip on any sense of whether there is a sharp line. I have no intuition, for example, that tells against a sharp line between the definitely definitely definitely definitely red and the not definitely definitely definitely definitely red. Accord with intuition cannot guide us in settling the issue; other virtues of the available theories must point the way. And one virtue of a precisificationalist theory which accepts a sharp line in the extreme case of an infinite stack of 'definitely' operators is that it allows us to avoid accepting weird objects like tower-noses. The appeal of such a theory is therefore undeniable, at least to commonsense moderates about composition, who like precisificational accounts but find tower-noses absurd.

The resulting theory is not what is normally thought of as an epistemic approach to vagueness. For while it does posit an unknowable sharp line between the superdefinitely red and the non-super-definitely red in a standard sorites series, it does not posit an unknowable sharp line between the red and non-red, the bald and non-bald. It is the latter sort of unknowable sharp line that I take to be intuitively problematic, as I have just explained. The theory also does not provide an epistemic analysis of vagueness, as epistemic theories do. It is only similar to epistemic theories in that it embraces a kind of unknowable sharp line. ${ }^{11}$

In any case, whether or not I have made a convincing case that this is the best version of precisificationalism, it seems clear that this version of precisificationalism is attractive on a number of counts and has not been ruled out. And so, contrary to Lewis, there are live versions of precisificationalism that are consistent with a commonsense moderate view of composition.

11 Williamson (ibid.) is the locus classicus for epistemic theories. 


\section{Third Objection: Explanatory Role of Composition}

One might respond to the foregoing by granting everything that I have said about what $I$ call 'composition'. But one might suggest that there has to be some other relation - call it shmomposition - which holds among the simples and the protocloud. And one might say that it is because the simples shmompose the protocloud that there is a further object in addition to the simples. And, because shmomposition is what explains the facts about existence we were interested in (e.g., facts about whether tower-noses exist), one might claim that shmomposition is the metaphysically interesting relation - the one that plays the metaphysically interesting explanatory role. If this view were right, I would have in effect changed the subject by focusing on the relation that I call 'composition'. And, were we to refocus the debate on the metaphysically interesting relation of shmomposition, commonsense moderates about composition would be caught in a dilemma: we would either be forced to admit that the metaphysically interesting composition relation - shmomposition - is sharply restricted, or we would finally have to admit that it is unrestricted. Since sharp restrictions do not serve any intuitions we have about any composition-like relation, however, restriction is (as Lewis puts it) gratuitous. Thus, according to this objection, composition in the metaphysically relevant sense (i.e., shmomposition) is unrestricted after all.

This approach is ideologically unparsimonious. For it is possible to define shmomposition, in my terms, as follows:

the xs shmompose $\mathrm{y} \leftrightarrow \sim$ (the $\mathrm{xs}$ are a super-definite case of noncomposition with respect to y).

And it seems, given the necessary truth of this biconditional, that the facts about the existence of composite objects can be explained at least as well by appeal to facts about composition in my sense (including facts about when it is vague whether composition occurs, and when it is vague whether it is vague, etc.). As a result, since my notion of composition is already needed to explain other intuitions, such as the intuition that it could be vague whether composition occurs, the most ideologically parsimonious approach is to accept the above biconditional as a definitional reduction of facts about shmomposition to facts about composition. Composition is in this sense a more fundamental relation than shmomposition, and thus is clearly of metaphysical interest, contrary to the present objection. 
However, someone might insist that facts involving the relation I call composition are not well suited to explain all of the relevant facts. For example, someone might say that, if the proto-cloud is located in the region which is the sum of the locations of the simples, the facts about the location (and also the shape and mass) of the proto-cloud are not well explained by the fact that the proto-cloud has the simples as borderline parts. For, they might point out, the fact that Kilimanjaro has $\mathrm{R}$ as a borderline part does not entail that Kilimanjaro is located in sum of the regions inhabited by Kilimanjaro and $\mathrm{R}$, since it is vague whether Kilimanjaro is located there.

My reply is that what explains the facts about the location of the proto-cloud is not the fact that it has the simples as borderline parts. Rather, the explanatory fact is that the proto-cloud has these simples as homogeneously borderline parts. (The xs are homogeneously borderline parts of $y$ iff the xs constitute a homogeneous case of borderline composition with respect to y.) I say that things inherit properties like mass and location from their homogeneously borderline parts. And so composition as I conceive of it is well suited to play the required explanatory role. $^{12}$

\section{References}

Hawley, Katherine. 2002. "Vagueness and Existence". Proceedings of the Aristotelian Society 102: 125-40.

Hawthorne, John. 2006. Metaphysical Essays. Oxford: Oxford University Press.

Koslicki, Kathrin. 2003. "The Crooked Path from Vagueness to FourDimensionalism". Philosophical Studies 114: 107-34.

Lewis, David. 1986. On the Plurality of Worlds. Oxford: Basil Blackwell. Markosian, Ned. 1998a. "Brutal Composition". Philosophical Studies 92: 211-49. Markosian, Ned. 1998b. "Simples". Australasian Journal of Philosophy 76: 21326.

Markosian, Ned. 2004. "Two Arguments from Sider's Four-Dimensionalism". Philosophy and Phenomenological Research 68: 665-73.

12 Thanks to Ralf Bader, George Bealer, Mark Crimmins, Dan Giberman, Cody Gilmore, Bernard Linsky, John Perry, Bryan Pickel, Adam Sennet, and audiences at Stanford, Davis, and the University of Alberta at Edmonton. Special thanks to Dan Korman for many hours of fruitful discussion, and to an anonymous referee for excellent comments that led to numerous improvements. 
McDaniel, Kris. 2007a. "Brutal Simples". In Dean Zimmerman, ed., Oxford Studies in Metaphysics, vol. 3.

McDaniel, Kris. 2007b. "Extended Simples". Philosophical Studies 133: 131-41.

Merricks, Trenton. 2005. "Composition and Vagueness". Mind 114: 615-37.

Nolan, Daniel. 2006. "Vagueness, Multiplicity, and Parts". Nous 40: 716-37.

Parsons, Josh. 2007. "Theories of Location." In Dean Zimmerman, ed., Oxford Studies in Metaphysics, vol. 3.

Scala, Mark. 2002. "Homogeneous Simples". Philosophy and Phenomenological Research 64: 393-7.

Sider, Ted. 2001. Four-Dimensionalism. Oxford University Press.

Sider, Ted. 2007. "Parthood". Philosophical Review 116: 51-91.

Smith, Donald. 2006. "The Vagueness Argument for Mereological Universalism”. Pacific Philosophical Quarterly 87: 357-68.

Van Inwagen, Peter. 1981. "The Doctrine of Arbitrary Undetached Parts". Pacific Philosophical Quarterly 62: 123-37.

Van Inwagen, Peter. 1990. Material Beings. Cornell University Press.

Williamson, Timothy. 1994. Vagueness. Routledge. 